\title{
Índices de capacidade de relações funcionais lineares e não-lineares
}

\author{
Edivaldo Antonio Bulba \\ Depto Eng. de Produção - USP \\ Coordenador de Metrologia Industrial na Faculdade de Engenharia Industrial \\ E-mail:edivaldo.bulba@poli.usp.br \\ LINDA LeE Ho \\ Depto. Eng. de Produção - USP \\ E-mail: lindalee@usp.br
}

\section{Resumo}

São apresentados métodos para determinação dos índices de capacidade tais como $C p$ e Cpk aplicados no "design for six sigma" (DFSS) quando a característica de qualidade é não-observável e dada por $Y=f\left(X_{1}, X_{2}, X_{3}, \ldots . X_{k}\right.$, onde $X$ são variáveis aleatórias com média e variância respectivamente iguais a $\mu_{X i} e \sigma_{X i}^{2} ; f$ é uma função conhecida. A determinação é baseada na série de Taylor e o procedimento será ilustrado com exemplos práticos.

\section{Palavras-chave}

Índice de capacidade de uma relação funcional, propagação dos erros, série de Taylor.

\section{Capability index for linear and non-linear functions}

\begin{abstract}
Here we present methods to determine capability indices such as Cp and Cpk to be applied in "design for six sigma" (DFSS) when the quality characteristic of interest $Y$ is unobservable but given by $Y=f\left(X_{1}, X_{2}, X_{3}, \ldots . X_{k}\right.$, where $X_{i}$ 's are random variables with means and variance respectively equal to $\mu_{X i}$ and $\sigma_{x i}^{2} ;$ is a known function. The determination is based on Taylor series and we illustrate the procedure with practical examples.
\end{abstract}

Key words

Capability index of a function, error propagation, Taylor series. 


\section{INTRODUĈ̣̃O}

Os Índices de capacidade foram introduzidos na década de 70 desde que Juran (1974) apresentou o pioneiro índice de capacidade $C p$. A suposição usual é que a característica de interesse seja observável com distribuição normal. Neste trabalho serão apresentados métodos baseados nas séries de Taylor para determinação dos índices de capacidade quando a característica de qualidade de interesse $Y$ não é observável mas dada por $Y=f\left(X_{1}\right.$, $X_{2}, X_{3}, \ldots . X_{k}$ ), onde $f$ é uma relação funcional de um certo número de variáveis de entrada independentes $Y=f\left(X_{l}\right.$, $\left.X_{2}, X_{3}, \ldots . X_{k}\right)$, sendo $X_{i}$ variável aleatória com $E\left(X_{i}\right)=\mu_{X i}$ e $\operatorname{Var}\left(X_{i}\right)=\sigma_{X i}^{2}$. Neste trabalho os índices de capacidade obtidos de uma relação funcional são empregados na fase de projeto, o que contribui para sistematizar e integrar as atividades de projeto e processo, garantindo a qualidade desde o projeto por estabelecer tolerâncias corretas (BULBA, 2003; BULBA; HO, 2002). Primeiramente encontram-se os pressupostos e as notações; a seguir fazse uma breve revisão destes índices; e apresentam-se alguns exemplos numéricos. Finalmente as conclusões são apresentadas.

\section{NOTAC̣ÕES E HIPÓTESES}

Para obter índices de capacidade de uma característica de qualidade não-observável, mas obtida por intermédio de uma relação funcional, foram consideradas as seguintes condições:

- as distribuições de probabilidade das variáveis de entrada e de saída são normais ou aproximadamente normais;

- conhece-se a relação funcional entre as variáveis de entrada e a variável de resposta (modelo univariado);

- em modelos não-lineares, as tolerâncias e variâncias das variáveis de entrada têm valores que propiciam uma linearização localizada, com erros desprezíveis;

- abordar-se-á a condição "nominal é melhor", quando a variabilidade e a tolerância são distribuídas simetricamente em torno do valor nominal.

As notações adotadas neste trabalho são:

$C p_{Y} \rightarrow$ índice de capacidade $C p$ para a variável de saída não-observável $Y$.

$C p k_{Y} \rightarrow$ índice de capacidade $C p k$ para a variável de saída não-observável $Y$.

$\mu_{Y}, \sigma_{Y} \rightarrow$ média e desvio-padrão da variável de saída nãoobservável $Y$.

$T_{Y} \rightarrow$ tolerância determinística de $Y$.

$\mu_{X i}, \sigma_{X i} \rightarrow$ média e desvio-padrão da variável de entrada $X_{i}$.

$m_{X i} \rightarrow$ valor nominal da variável de entrada $X_{i}$.

$\mathrm{T}_{X i} \rightarrow$ tolerância determinística de $X_{i}$.

\section{ÍNDICES DE CAPACIDADE DE UMA RELAC̣ÃO FUNCIONAL CP $_{\mathrm{Y}}$ E CPK $_{\mathrm{Y}}$}

O índice de capacidade " $C p$ " foi definido por Juran (1974) como: $C p=\frac{T}{6 \sigma}$ onde $T$ é a tolerância de projeto e $6 \sigma$ é a tolerância natural do processo (BANKS, 1989; BURR, 1976). No caso de várias variáveis de entrada, $X_{i}, i=$ $1,2,3, \ldots . . k$, que definem um modelo matemático $f_{Y}=\left(X_{l}\right.$, $\left.X_{2}, X_{3}, \ldots . X_{k}\right)$, onde $E\left(X_{i}\right)=\mu_{X i}$ e $\operatorname{Var}\left(X_{i}\right)=\sigma_{X i}^{2}, \mathrm{i}=1, \ldots, \mathrm{k}$, o índice de capacidade $C p$ da variável $Y$ é dado pela relação:

$$
C p_{Y}=\frac{T_{Y}}{6 \sigma_{Y}}
$$

onde

$$
T_{Y} \cong \sum_{i=1}^{k}\left|\frac{\partial f}{\partial X_{i}}\right| \mathbf{x}=\mu \mid T_{X i}
$$

expressa a tolerância acumulada de projeto na variável de resposta $Y$ com $\mathbf{X}=\left(\mathrm{X}_{1}, \ldots, \mathrm{X}_{\mathrm{k}}\right)$ e $\boldsymbol{\mu}=\left(\mu_{\mathrm{x} 1}, \ldots, \mu_{\mathrm{xk}}\right)$. Observese que esta tolerância acumulada é determinística e é obtida pela somatória das contribuições das tolerâncias das variáveis de entrada $T_{X i}$ em $T_{Y}$. Cada contribuição é proporcional à tolerância $T_{X i}$ e sua derivada parcial em valor absoluto (CREVELING, 1997). Esta somatória corresponde a uma combinação linearizada por meio da aproximação pela primeira ordem da série de Taylor. Por sua vez, $6 \sigma_{Y}$ é a tolerância natural de processo, sendo que $\sigma_{Y}$ é aproximado por:

$$
\sigma_{Y} \cong \sqrt{\sum_{i=1}^{K}\left(\frac{\partial f}{\partial X_{i}} \mid \mathbf{x}=\mu\right)^{2} \sigma_{X_{i}}^{2}+2 \sum_{i=1}^{K-1} \sum_{j=i+1}^{K} \frac{\partial f}{\partial X_{i}} \frac{\partial f}{\partial X_{j}} \sigma_{X_{i}} \sigma_{X_{j}} \rho_{X_{i}, X_{j}}}
$$

onde $\rho x i, x j$ é o coeficiente de correlação entre $X_{i}$ e $X_{j}$. Quando $X_{i}$ e $X_{j}$ são independentes, a segunda parcela dentro do radical da expressão (3) é omitida. É importante salientar que uma combinação linear de variáveis de entrada com distribuições normais, independentes $\boldsymbol{o u}$ ñ̃o resulta em uma variável de resposta $Y$ também com distribuição normal (DIETRICH, 1991). Assim, devido à normalidade da variável de resposta, os índices de capacidade de uma relação funcional podem ser definidos. Deve-se ressaltar que a expansão de Taylor até a primeira ordem somente pode ser aplicada quando os erros de segunda ordem forem desprezíveis; não obstante, Ullman (1997) destaca que esta condição é alcançada na 
maioria dos casos práticos.

Na Figura 1 está uma representação gráfica e analítica do índice de capacidade $C p$ de uma variável $Y$ obtido a partir de duas variáveis independentes.

As tolerâncias das variáveis de entrada $T_{X 1}$ e $T_{X 2}$ estão representadas respectivamente pelos segmentos $B X_{1} C X_{1}$ e $B X_{2} C X_{2}$. A contribuição destas tolerâncias para a variável de resposta $Y$ é obtida pela técnica de linearização e é dada aproximadamente, pelos segmentos:

$A X_{1} B X_{1}=\left|\frac{\partial f}{\partial X_{1}}\right| \mathbf{x}=\mu \mid T_{X 1}$ e $A X_{2} B X_{2}=\left|\frac{\partial f}{\partial X_{2}}\right| \mathbf{x}=\mu \mid T_{X 2}$.

A tolerância $T_{Y}$ está representada pelo segmento $A Y B Y=A X_{1} B X_{1}+A X_{2} B X_{2}$ que corresponde a:

$T_{Y}=\left|\frac{\partial f}{\partial X_{1}}\right| \mathbf{x}=\mu\left|T_{X 1}+\right| \frac{\partial f}{\partial X_{2}}|\mathbf{x}=\mu| T_{X 2}$. Para ilustração, considerar-se-á, por simplicidade, $C P_{X 1}=C P_{X 2}=1$, sem perda de generalidade. Segue que $T_{x 1} \cong 6 \sigma_{x 1}$ e $T_{x 2} \cong 6 \sigma_{x 2}$. Desta forma, a tolerância natural na resposta será:

$$
A^{\prime} Y B^{\prime} Y=6 \sigma_{Y} \cong \sqrt{\left(\left(\frac{\partial f}{\partial X_{1}}\right) 6 \sigma_{X 1}\right)^{2}+\left(\left(\frac{\partial f}{\partial X_{2}}\right) 6 \sigma_{X 2}\right)^{2}}
$$

e o índice de capacidade de $Y: C P_{Y}=\frac{T_{Y}}{6 \sigma_{Y}}=\frac{A Y B Y}{A^{\prime} Y B^{\prime} Y}$. Note que $A Y B Y>A^{\prime} Y B^{\prime} Y$, portanto, neste caso, é maior do que 1 . Isto é devido à soma quadrática das tolerâncias naturais de entrada.

Similarmente, pode-se obter o índice de capacidade de uma relação funcional $C p k$ para a variável de resposta $Y$ $\left(C p k_{y}\right)$ que expressa concomitantemente erros de precisão ou aleatórios (variabilidade medida pelo desviopadrão $\sigma$ ) e erros de posicionamento ou sistemáticos (expressos pela média $\mu$ ). Seja:

$$
k=\frac{2|\mu-m|}{(L S E-L I E)}
$$

Figura 1: Acúmulo de tolerâncias num modelo não-linear com duas variáveis de entrada.

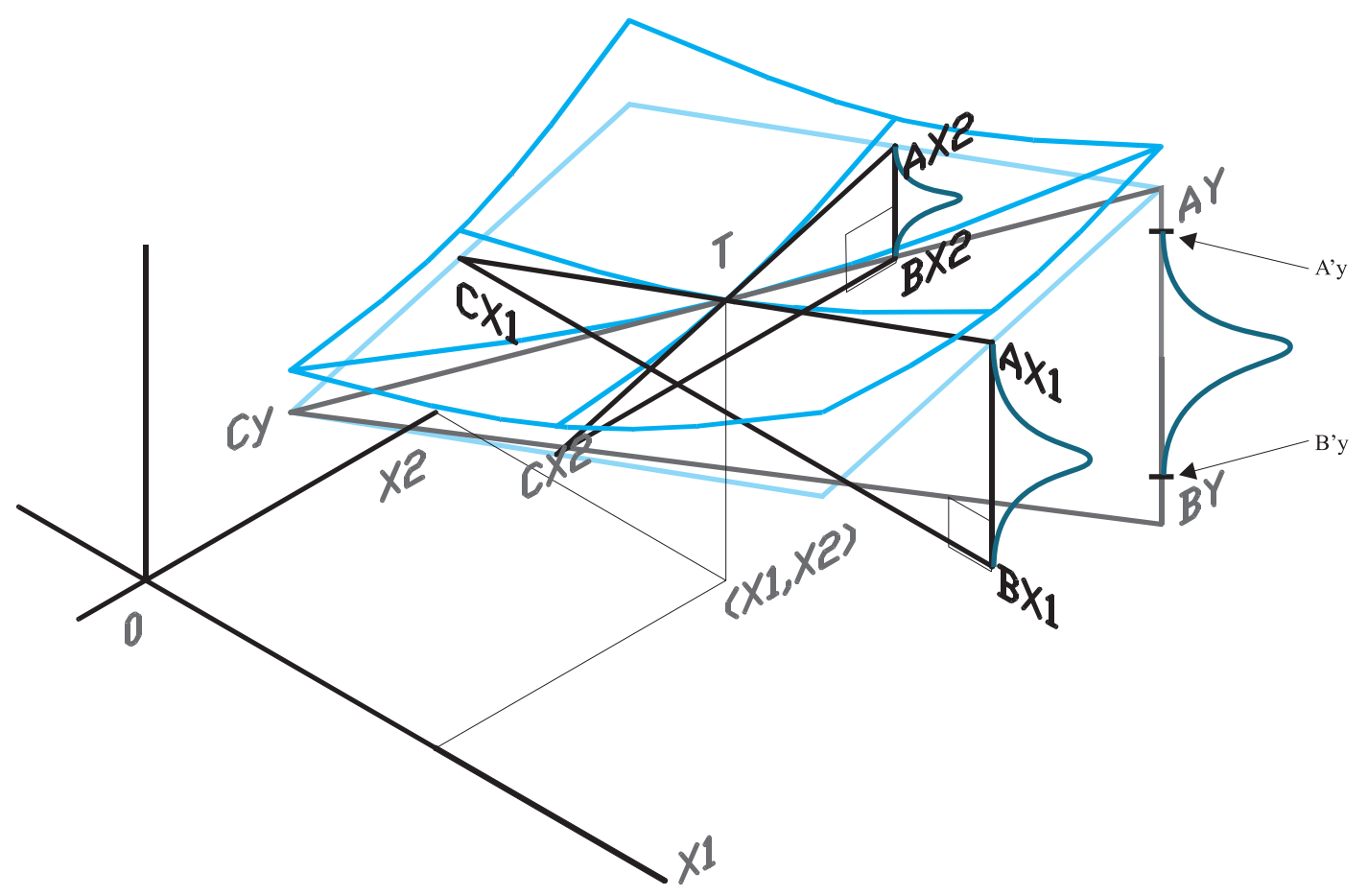


onde $m$ é o valor central de projeto, $|\mu-m|$ corresponde ao erro sistemático absoluto, LIE e LSE são respectivamente os limites inferior e superior da especificação, a diferença $(L S E-L I E)$ corresponde à tolerância de projeto e o fator $k$ expressa quanto o processo está descentralizado para determinado lado da especificação. Por exemplo, $k=0,25$ corresponde a uma descentralização de $25 \%$ para a direita ou para a esquerda. Segue que os índices se relacionam através de

$$
C p_{k}=C p(1-k)
$$

Substituindo (4) em (5) a relação fica:

$$
C p_{k}=\min \left(\frac{\mu-L I E}{3 \sigma} ; \frac{L S E-\mu}{3 \sigma}\right)
$$

A importância de se prever erros sistemáticos envolvendo acúmulo de tolerâncias antes de ocorrer a manufatura (projeto dos processos) foi abordada por Evans (1975), que propõe um fator de segurança de 1,5 no desvio-padrão da variável de resposta; assim, segundo esta abordagem, obtemos um $C p_{Y}$ com margem de segurança substituindo o valor de $C p k_{Y}$. Já outros autores, como Breyfogle (1999) e Harry (1987), estabelecem um erro sistemático e simétrico nas variáveis de entrada correspondente a $\pm 1,5 \sigma$ em torno da média como modo de se levarem em conta os erros sistemáticos nas circunstâncias envolvendo acúmulo de tolerâncias. Wilson (2000), entretanto, critica este procedimento, argumentando que o erro sistemático não pode ser fixado arbitrariamente em $\pm 1,5 \sigma$. Portanto, um procedimento é apresentado no presente trabalho onde se pode estabelecer no projeto qualquer valor de erro sistemático simétrico para cada variável de entrada, o que conduzirá ao valor do erro sistemático na variável de resposta. Para obter o fator $k$ da variável $Y$ deve-se novamente aplicar a expansão da primeira ordem da série de Taylor junto aos erros sistemáticos estabelecidos para as variáveis de entrada. O erro sistemático simétrico confere um comportamento semelhante ao do acúmulo das tolerâncias. Devido a isto, as diferenciais parciais na expansão de Taylor devem aparecer em valor absoluto e os erros sistemáticos ou de posição de cada variável também. Assim:

$$
C p k_{Y}=C p_{Y}\left(1-k_{Y}\right)
$$

onde $C p_{Y}$ é dado em (1); $k_{Y}=\frac{\left|\mu_{Y}-m_{Y}\right|}{T_{Y}}$ e $\left|\mu_{Y}-m_{Y}\right|$ corresponde ao erro sistemático da variável de resposta, expresso por:

$$
\left|\mu_{Y}-m_{Y}\right|=\sum_{i=1}^{k}\left|\mu_{X i}-m_{X i}\right|\left|\frac{\partial f}{\partial X_{i}}\right| \mathbf{x}=\mu \mid
$$

\section{EXEMPLOS NUMÉRICOS}

\section{Exemplo 1 - Controle da área de um retângulo}

Considere uma produção de chapas retangulares cortadas na largura $X_{1}$ e no comprimento $X_{2}$, onde a área resultante é uma característica crítica de qualidade que deve ter o padrão "seis sigma", ou seja $C p k \geq 2$. Veja a Figura 2.

Figura 2: Exemplo de controle da área de uma chapa retangular.

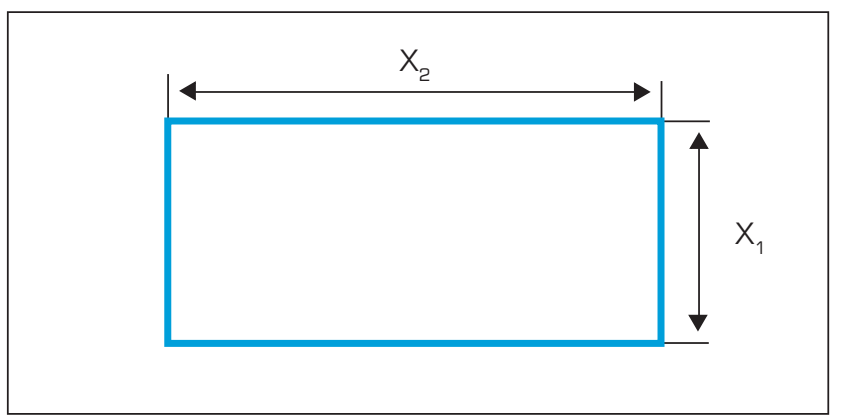

Os valores médios, tolerâncias e diferenciais parciais de $X_{1}$ e $X_{2}$ são respectivamente:

$$
\begin{array}{lll}
\mu_{X 1}=100 \mathrm{~mm} & T_{X 1}=0,2 & \frac{\partial f}{\partial X_{1}}=200 \\
\mu_{X 2}=200 \mathrm{~mm} & T_{X 2}=0,2 & \frac{\partial f}{\partial X_{2}}=100
\end{array}
$$

A função que relaciona a variável de resposta $Y$ e as variáveis de entrada é: $Y=X_{1} . X_{2}$. Para determinar a tolerância da variável de resposta, aplica-se (2):

$$
\begin{aligned}
& T_{Y}=\left|\frac{\partial f}{\partial X_{1}}\right| \mathbf{x}=\mu\left|T_{X 1}+\right| \frac{\partial f}{\partial X_{2}}|\mathbf{x}=\mu| T_{X 2}= \\
& 200.0,2+100.0,2=60
\end{aligned}
$$

Para obter $C p k_{y} \geq 2,0$, estabelecem-se valores de desvios-padrão das variáveis de entrada que garantirão o nível de qualidade requerido na variável de resposta. Neste caso, após análises envolvendo processos disponí- 
veis e custos, foram estabelecidos $\sigma_{x 1}=\frac{T_{x 1}}{8}$ e $\sigma_{x 2}=\frac{T_{x 2}}{10}$, que resultam respectivamente $C p_{X 1}=1,67$ e $C p_{X 2}=1,33$. Aplicando-se (3):

$$
\sigma_{Y} \cong \sqrt{\left(\frac{\partial f}{\partial X_{1}} \mid \mathbf{x}=\mu\right)^{2} \sigma_{X 1}^{2}+\left(\frac{\partial f}{\partial X_{2}} \mid \mathbf{x}=\mu\right)^{2} \sigma_{X 2}^{2}}=\sqrt{200^{2}\left(\frac{0,2}{8}\right)^{2}+100^{2}\left(\frac{0,2}{10}\right)^{2}}=4,125
$$

E o índice de capacidade da variável de resposta, $C p_{Y}$ (1) será igual a :

$$
C p_{Y}=\frac{T_{Y}}{6 \sigma_{Y}}=\frac{60}{24,75}=2,42
$$

De modo similar, aplicando (6), a tolerância do erro sistemático da variável de resposta é determinada. Dados: $\left|\mu_{x 1}-m_{x 1}\right|=0,01$ e $\left|\mu_{x 2}-m_{x 2}\right|=0,02$, segue que:

$$
\begin{aligned}
\left|\mu_{Y}-m_{Y}\right| & =\left|\mu_{X 1}-m_{X 1}\right|\left|\frac{\partial f}{\partial X_{1}}\right| \mathbf{x}=\mu|+| \mu_{X 2}-m_{X 2}|| \frac{\partial f}{\partial X_{2}}|\mathbf{x}=\mu| \\
& =|0,01| \cdot|200|+|0,02| \cdot|100|=4
\end{aligned}
$$

Segue que o fator $k_{y}$ da variável de resposta $Y$ é dado por:

$$
k_{Y}=\frac{\left|\mu_{Y}-m_{Y}\right|}{T_{Y} / 2}=\frac{|4|}{60 / 2}=0,133
$$

E o índice $C p k_{Y}=C p_{Y}\left(1-k_{Y}\right)=2,42(1-0,133)=2,1$

\section{Exemplo 2 - Reavaliação de tolerâncias de projeto}

Devido à dificuldade em trabalhar com relações nãolineares, alguns deixam de "linearizar" determinadas funções não-lineares, através da aplicação da primeira ordem da série de Taylor. Desta forma consideram a relação entre variáveis originalmente como linear, ao aplicar uma simples soma de tolerâncias ou de variâncias a fim de estimar ou prever a tolerância ou a variância na variável de resposta. Segundo Creveling (1997), este procedimento pode ser

Figura 3: Exemplo de modelo não-linear.

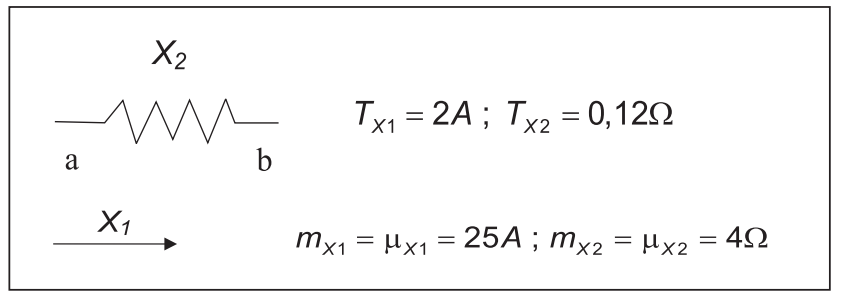

prejudicial. Para ilustrar este fato, considere um exemplo em Montgomery (2001). Num circuito simples em corrente contínua, a tensão $Y$ entre dois pontos $a$ e $b$ deve ser de $100 \mathrm{~V}$ com $T_{y}=4 \mathrm{~V}$. A corrente e a resistência do circuito, especificações das variáveis de entrada, são respectivamente:

Assume-se neste caso que as variáveis $X_{1}$ e $X_{2}$ são normalmente distribuídas e independentes entre si, com médias coincidindo com os valores nominais, de modo que a variável de resposta $Y$ também tenha uma distribuição normal. Ainda, considera-se a previsão das tolerâncias naturais de processo de $X_{1}$ e $X_{2}$ como coincidentes com as respectivas especificações-limite em $99,7 \%(\mu \pm 3 \sigma)$, ou seja:

$$
\begin{aligned}
& C p_{x 1}=C p k_{x 1}=C p_{x 2}=C p k_{x 2}=1 \Rightarrow \sigma_{x 1}=1 / 3=0,33 \\
& \text { e } \sigma_{x 2}=0,06 / 3=0,02
\end{aligned}
$$

Pela lei de Ohm, $Y=X_{1} X_{2}$. Nestas condições, a média de $Y$ será aproximadamente: $\mu_{Y} \cong \mu_{X 1} \cdot \mu_{X 2}=25,4=100$. Como $\frac{\partial f}{\partial X_{1}}=X_{2}$ e $\frac{\partial f}{\partial X_{2}}=X_{1}$, segue que o desvio-padrão de $Y$ calculado no ponto $\left(\mu_{x_{1}}, \mu_{x_{2}}\right)$ é dado por:

$$
\begin{aligned}
& \sigma_{Y}^{2} \cong\left(\frac{\partial f}{\partial X_{1}} \mid \mathbf{x}=\mu\right)^{2} \sigma_{X 1}^{2}+\left(\frac{\partial f}{\partial X_{2}} \mid \mathbf{x}=\mu\right)^{2} \sigma_{X 2}^{2} \Rightarrow \\
& \sigma_{Y}^{2} \cong \mu_{X 2}^{2} \cdot \sigma_{X 1}^{2}+\mu_{X 1}^{2} \sigma_{X 2}^{2} \\
& \sigma_{Y}^{2} \cong 4^{2} \cdot 0,33^{2}+25^{2} \cdot 0,02^{2} \cong 2,00 \\
& \sigma_{Y} \cong \sqrt{2,00} \cong 1,41
\end{aligned}
$$

Considerando os limites naturais de variação de tensão $Y$, tem $6 \sigma_{Y}=8,46 \mathrm{~V}$. No entanto, esta variação de $Y$ excede à especificação inicial $T_{Y}=4 \mathrm{~V}$, que resulta em um índice de capacidade sofrível:

$$
C p_{Y} \cong \frac{T_{Y}}{6 . \sigma_{Y}} \cong \frac{4,0}{6 \cdot 1,41} \cong \frac{4,0}{8,46} \cong 0,47
$$

Isto se dá devido ao acúmulo de variabilidade e a uma escolha equivocada de tolerâncias, por não considerarem as relações não-lineares entre as variáveis de entrada. Tolerâncias supostamente aceitáveis nas variáveis de entrada sem levar em consideração as relações não-lineares não refletirão uma tolerância adequada da variável de resposta. Para evitar este problema, os valores das tolerâncias e variân- 
cias das variáveis de entrada serão reavaliados com o objetivo de adequar o valor da tolerância da variável de resposta (BURR, 1976 e CREVELING, 1997). A lei geral de propagação do erro deve ser aplicada com o objetivo de en-

$$
C p_{Y} \cong \frac{\left|\frac{\partial f}{\partial X_{1}}\right| \mathbf{x}=\mu\left|T_{X 1}+\right| \frac{\partial f}{\partial X_{2}}|\mathbf{x}=\mu| T_{X 2}}{6 \cdot \sqrt{\left(\frac{\partial f}{\partial X_{1}} \mid \mathbf{x}=\mu\right)^{2} \sigma_{X 1}^{2}+\left(\frac{\partial f}{\partial X_{2}} \mid \mathbf{x}=\mu\right)^{2} \sigma_{X 2}^{2}}} \cong \frac{4 \cdot 0,727+25.0,044}{6 \cdot \sqrt{4^{2} \cdot 0,117^{2}+25^{2} \cdot 0,007^{2}}} \cong 1,33
$$

Uma vez que se respeitou a relação não-linear do índice de capacidade $C p_{Y}$ da tensão, coerentemente este se confirmou em 1,33 a partir dos valores redefinidos de tolerância.

\section{CONCLUSÕES}

Os índices de capacidade de uma relação funcional $C p_{Y}$ e $C p k_{Y}$ proporcionam um melhor controle de qualidade sobre uma característica de qualidade que seja variável de resposta dependente de várias variáveis de entrada. Ressalta-se que o procedimento proposto neste trabalho é empregado na fase de projeto dos processos, contribuindo diretamente para o "design for six sigma". A previsão de índices de capacidade no projeto é um meio de garantir a qualidade desde a concepção do produto, propiciando uma seleção mais racional dos processos de manufatura a serem empregados. Posteriormente, na manufatura, podemse utilizar as estimativas dos desvios-padrão de $\sigma_{X i}$ e das médias $\mu_{X i}$ fornecidas pelo controle estatístico de processo; desta forma, estimativas pontuais e intervalares de $C p_{Y}$ e $C p k_{Y}$ podem ser obtidas, porém esta não é a abrangência deste trabalho. Ressalta-se que a relação entre as variáveis de entrada pode ser linear ou não. Quando o modelo é linear, os índices $C p_{Y}$ e $C p k_{Y}$ são exatos; quando o modelo matemático não é linear, aplica-se uma linearização através da expansão de Taylor limitada à primeira ordem, o que na grande maioria dos casos revela-se suficiente.

\section{Artigo recebido em 16/08/2003 Aprovado para publicação em 03/02/2004}

\section{- Bibliografia}

BANKS J. Principles of Quality Control, New York, John Wiley \& Sons, First Edition, Georgia Institute of Technology, 1989.

BREYFOGLE III, F. W. Implementing Six Sigma - New York, Ed. John Wiley \& Sons, 1999.

BULBA E. A. Contribuições ao Estudo de Índices de Capacidade de uma Relação Funcional. São Paulo, Tese de Doutorado. EPUSP. 2003.
BULBA E. A.; Ho L. L. Índices de Capacidade Combinados. Curitiba, ENEGEP 2002.

BURR, I. W. Statistical Quality Control Methods, New York, Marcel Dekker, 1976. CREVELING, C. M. Tolerance Design, Massachusetts, Addison Wesley Longman, Inc, 1997.

Dietrich, C. F. Uncertainty, Calibration and Probability, New York, Adam Hilger, Second Edition, 1991.
EVANS, D.H. Statistical Tolerancing: The State of the Art, Part III. Shifts and Drifts. Journal of Quality Technology, v. 7, n. 2, 1975.

HARRY, M.; STEWART R. Six Sigma Mechanical Design Tolerancing, Motorola University Press, Schaumburg, IL, 1988.

JURAN, J. M. Quality Control Handbook, New York, Mc Graw-Hill, 1974.
MONTGOMERY, D. C Introduction to Statistical Quality Control. Cingapura, Ed. John Wiley \& Sons, Fourth Edition, 2001.

ULLMAN, D. G. The Mechanical Design Process, Oregon State University, Second Edition, Mc Graw-Hill International Editions, 1997.

WILSON, M.P. Seis Sigma-Compreendendo o Conceito, As Implicações e os Desafios. Rio de Janeiro, Ed. Qualitymark, 2000.

\section{- Agradecimentos}

Os autores agradecem os revisores deste trabalho pelas sugestões que sem dúvida contribuíram para o enriquecimento do mesmo. 